\title{
Dor, Autorregulação e Temperamento em Recém-nascidos Pré-termo de Alto Risco
}

\author{
Pain, Self-Regulation and Temperament in High Risk Preterm Newborns \\ Vivian Caroline Klein, Cláudia Maria Gaspardo, \& Maria Beatriz Martins Linhares* \\ Universidade de São Paulo, Ribeirão Preto, Brasil
}

\begin{abstract}
Resumo
Os recém-nascidos pré-termo são expostos a experiências dolorosas inevitáveis na Unidade de Terapia Intensiva Neonatal. Apesar de sua imaturidade biológica, esses podem tanto perceber quanto reagir à dor. A exposição repetida à dor exerce impacto negativo no desenvolvimento e pode aumentar a vulnerabilidade dos bebês, dificultando os processos autorregulatórios. A reatividade biocomportamental à dor é um indicador de autorregulação e associa-se ao temperamento da criança. Esses bebês necessitam de estratégias farmacológicas e não farmacológicas para alívio da dor. Além disso, devem ter um contexto de interações sincrônicas com os pais, adaptadas às suas características de temperamento. Os profissionais de saúde podem atuar como suporte de regulação externa para esses bebês promovendo alívio da dor e prevenindo problemas de desenvolvimento.
\end{abstract}

Palavras-chave: Dor; Nascimento Prematuro; Reatividade-estabilidade; Temperamento.

\begin{abstract}
High risk preterm newborns are exposed to unavoidable painful experiences in Neonatal Intensive Care Units. In spite of their biological immaturity, they are able to perceive and react to pain stimuli. Repetitive exposure to pain has a negative impact on the development and may increase the infant's vulnerability, affecting self-regulatory processes. Biobehavioral reactivity to pain is an indicator of self-regulation and it is associated to the child's temperament. Preterm infants need pharmacological and non-pharmacological strategies for pain relieving. Moreover, they need a context of synchronic interaction with their parents which must be adapted to their temperament characteristics. Health professionals may play an important role in supporting external regulation for those infants, promoting pain relief and preventing development problems.
\end{abstract}

Keywords: Pain; Premature Birth; Reactivity-stability; Temperament.

\section{O Recém-Nascido Pré-Termo}

O nascimento pré-termo $(<37$ semanas de idade gestacional), com peso abaixo de 1.500 gramas, é identificado como um fator de risco que ameaça a trajetória de desenvolvimento adaptativo da criança (Linhares, 2009; Minde, 2000). Na abordagem teórico-conceitual da Psicopatologia do Desenvolvimento (Luthar, Sawyer, \& Brown, 2006), fatores de risco consistem em variáveis do indivíduo, do ambiente, ou resultantes da combinação entre ambos, que aumentam a probabilidade de o indivíduo apresentar problemas de desenvolvimento.

\footnotetext{
* Endereço para correspondência: Universidade de São Paulo, Faculdade de Medicina de Ribeirão Preto, Laboratório de Pesquisa em Prevenção de Problemas de Desenvolvimento e Comportamento da Criança, salas 52/ 53, Avenida Tenente Catão Roxo, 2650, Prédio da Saúde Mental, Campus Monte Alegre, Ribeirão Preto, SP, Brasil, CEP 14049-900. Tel.: +55- 16- 3602-4610 / 36372702; Fax: +55- 16-3602-4607/ 3602-4504. E-mail: linhares@fmrp.usp.br

Agradecimentos: Fundação de Amparo à Pesquisa do Estado de São Paulo (FAPESP) e Conselho Nacional de Desenvolvimento Científico e Tecnológico (CNPq).
}

No Brasil, os últimos dados estatísticos disponíveis do Sistema Único de Saúde, referente ao ano-base de 2006, mostram um índice de 6,7\% de nascimentos pré-termo (197.310 bebês) em um total de 2.944.928 bebês nascidos vivos (Ministério da Saúde, 2009). Avanços significativos têm sido realizados quanto aos índices de sobrevivência de neonatos pré-termo de alto risco, atingindo taxas bem sucedidas, em torno de 50 a $90 \%$ em neonatos de 24 a 28 semanas de idade gestacional (Lorenz, 2000).

A trajetória de desenvolvimento do bebê pré-termo fora da vida uterina inicia-se no contexto hospitalar de uma Unidade de Terapia Intensiva Neonatal (UTIN) devido à necessidade de receber cuidados intensivos para sobreviver. O recém-nascido pré-termo apresenta-se imaturo, desorganizado e desintegrado em várias funções e sistemas do organismo relevantes para o seu desenvolvimento (Glass, 1999), ficando exposto a experiências atípicas de privação ou excesso de estímulos em um período sensível e ótimo para o crescimento cerebral rápido e diferenciação neuronal (Couperus \& Nelson, 2006).

No período pós-natal, são freqüentes as enfermidades crônicas, as quais podem ocasionar hospitalizações pro- 
longadas e repetidas. Embora a UTIN não faça mais parte do ambiente da criança, as repercussões da experiência da internação na UTIN para o bebê e sua família podem permanecer no contexto de desenvolvimento da criança, regulando as interações sociais entre mãe e criança (Klein \& Linhares, 2006; Landry, Smith, Swank, \& MillerLoncar, 2000; Laucht, Esser, \& Schmidt, 2002; Linhares, Martins, \& Klein, 2004).

Estudos que focalizam as trajetórias de desenvolvimento de crianças que nasceram pré-termo e com muito baixo peso revelam que, quando comparadas às crianças nascidas a termo, aquelas são mais propensas a apresentar os seguintes problemas: atraso neuro-comportamental (Barbosa, Formiga, \& Linhares, 2007), problemas cognitivos (Landry et al., 2000; Luu et al., 2009; Méio, Lopes, \& Morsch, 2003; Wolke, Samara, Bracewell, \& Marlow, 2008), dificuldades de linguagem (Luu et al., 2009; Wolke et al., 2008), problemas de desempenho escolar (Salt \& Redshaw, 2006; Wolke et al., 2008) e dificuldades comportamentais (Minde, 2000; Samara, Marlow, \& Wolke, 2008). Entre os processos desenvolvimentais aos quais as crianças nascidas pré-termo encontram-se mais vulneráveis, destacam-se a autorregulação (Clark, Woodward, Horwood, \& Moor, 2008; Feldman, 2009), a atenção (Luu et al., 2009) e a função executiva (Sun, Mohay, \& O’Callaghan, 2008).

Apesar de as crianças nascidas pré-termo apresentarem maior probabilidade para exibir problemas de desenvolvimento, trajetórias desenvolvimentais adaptativas também podem ser desencadeadas. É possível identificar nessas trajetórias a presença de mecanismos de proteção, que consistem em variáveis que podem ser atributos disposicionais do indivíduo, aspectos do meio ambiente ou da interação entre esses que modificam os efeitos adversos dos fatores de risco desencadeando desfechos adaptativos (Luthar et al., 2006).

Dessa forma, indivíduos podem apresentar desenvolvimento psicológico adaptado apesar de terem sido expostos a experiências adversas que se constituiriam em riscos potenciais para o desencadeamento de graves sequelas. Essas trajetórias resultantes em desenvolvimento positivo na presença dos fatores de risco envolvem processos de resiliência. Esta consiste em um processo dinâmico por meio do qual as adaptações positivas podem ser alcançadas em contexto de adversidade (Luthar et al., 2006; Yunes \& Zymanski, 2001). O conceito de resi-liência refere-se à combinação entre experiências adversas/negativas e desenvolvimento psicológico posterior positivo, apesar de experiências desta natureza. É considerado um conceito dinâmico, pois é inferido a partir do interjogo entre os fatores de risco e os mecanismos de proteção (Rutter, 2006). Luthar et al. (2006) destacam que a resiliência só pode ser alcançada e mantida por meio de relacionamentos fortes e sustentáveis com outras pessoas. Durante a infância, o relacionamento com os cuidadores primários afeta diversos atributos psicológicos emergentes e influencia o enfrentamento das princi- pais tarefas evolutivas; o cumprimento dessas tarefas, por sua vez, afeta a probabilidade de sucesso no enfrentamento das tarefas posteriores.

De acordo com a abordagem da Psicopatologia do Desenvolvimento, deve-se compreender as desordens de adaptação do indivíduo focalizando os intercâmbios probabilísticos entre dinâmicas de comportamento dos indivíduos e dos contextos ambientais, sem estabelecer predições deterministas, isomórficas e relações de causaefeito (Rutter, 2006; Sameroff, 2009).

\section{O Contexto Inicial do Desenvolvimento e a Dor em Bebês Nascidos Pré-termo}

Ao mesmo tempo em que o ambiente da UTIN constitui-se em cuidado especial e proteção para o bebê nascido pré-termo, também envolve múltiplos estímulos que provocam dor, estresse e desconforto, tanto para o bebê quanto para os seus familiares. A adversidade ambiental da UTIN para o bebê caracteriza-se por frequentes procedimentos médicos e manuseios invasivos e dolorosos, como por exemplo, repetidas punções do tipo arterial, venosa ou capilar para coleta de sangue, aspiração endotraqueal, intubação para ventilação mecânica e excesso de estimulação luminosa e sonora (Grunau, Holsti, \& Peters, 2006). Além disso, a internação na UTIN leva o bebê a ter certas privações, como as restrições aos contatos físicos prazerosos de contato pele-a-pele com a mãe e de amamentação. O sistema sensório-motor é relevante para a organização hierárquica e integrada do bebê (Als et al., 2004). A exposição aos estímulos provenientes do ambiente extrauterino desencadeia respostas relacionadas aos processos de autorregulação fisiológica, que consiste em processos e funcionamentos do sistema nervoso parassimpático. Esses exercem papel na etiologia dos comportamentos regulatórios iniciais que sustentam vínculos sociais, como o estado de vigília e sono, a atividade motora e a emoção (Feldman, 2009). O controle do alerta fisiológico se integra aos processos de atenção (Calkins, 2009). Dessa forma, a organização comportamental dos estados de vigília e sono dos bebês constitui-se em um índice neurofisiológico predominante de maturidade do sistema nervoso central (Feldman, 2009; Glass, 1999).

A estimulação aversiva no desenvolvimento inicial do bebê torna-se sobremaneira preocupante, considerandose que bebês prematuros, com menos de 32 semanas de idade gestacional, passam na UTIN a maior parte do tempo que corresponderia ao terceiro trimestre da gestação. Exatamente neste período em que ocorre acelerado desenvolvimento cerebral (Couperus \& Nelson, 2006), o vulnerável sistema nervoso central do bebê está exposto à dor e ao estresse.

A experiência de dor é considerada uma das principais condições de estresse para os neonatos pré-termo durante sua internação na UTIN (Anand \& Whit Hall, 2007; Grunau et al., 2006; Klein, 2009; Linhares, 2009). Neonatos com menos de 32 semanas de idade gestacional 
podem receber, como parte necessária ao seu tratamento médico para assegurar sua sobrevivência, aproximadamente seis procedimentos dolorosos por dia nas primeiras semanas de internação na UTIN, podendo chegar a 18 procedimentos dolorosos por dia (Gaspardo, Chimello, Cugler, Martinez, \& Linhares, 2008).

Devido à coleta de sangue ser o procedimento doloroso mais frequentemente realizado na rotina da UTIN, os estudos na área de reações à dor de bebês pré-termo tem observado as reações dos bebês neste tipo de procedimento. O Grupo de Controle de Dor Neonatal destaca que a inabilidade de comunicar verbalmente ou não verbalmente a dor não pode negar a possibilidade de que um indivíduo esteja experimentando dor e, portanto, precise de tratamento apropriado para aliviá-la (Anand et al., 2006).

Os bebês inserem-se na categoria de indivíduos que apresentam limitações para expressar a dor que sentem. No entanto, os bebês exibem respostas bioquímicas, fisiológicas e comportamentais em reação a procedimentos dolorosos (Anand et al., 2006; Franck \& Miakowski, 1997). Embora as vias transmissoras do estímulo doloroso não estejam ainda mielinizadas no feto durante o segundo e o terceiro trimestre de gestação, sendo completamente mielinizadas entre 30 a 37 semanas, as fibras nervosas são capazes de conduzir os estímulos dolorosos. A mielinização incompleta implica apenas velocidade de condução lenta no trajeto do sistema nervoso central. No entanto, no recém-nascido, o impulso nervoso percorre uma trajetória de curta distância, o que acaba compensando essa lentidão da transmissão do estímulo. Dessa forma, recém-nascidos prematuros já possuem capacidade neurológica suficiente para perceber a dor em nível cortical (Bartocci, Bergqvist, Lagercrantz, \& Anand, 2006; Slater, Fitzgerald, \& Meek, 2007).

Os bebês podem perceber a dor mais intensamente quando comparados a crianças mais velhas ou a adultos; isso se explica devido aos mecanismos de controle inibitório que são imaturos e tem limitações em sua sensibilidade para modular a experiência dolorosa. A imaturidade do sistema nervoso central do bebê pré-termo resulta, portanto, em dificuldades de enfrentamento de eventos de dor e estresse (Als et al., 2004).

Neonatos pré-termo, assim como fetos por volta de 18 a 20 semanas de idade gestacional, já apresentam reações fisiológicas e comportamentais à dor e ao estresse (Anand \& Whit Hall, 2007; Van de Velde, Jani, De Buck, \& Deprest, 2006). Anteriormente, havia a concepção de que a reação à dor em neonatos pré-termo eram respostas reflexas que ocorriam no nível do tronco cerebral. Nos últimos anos, estudos têm demonstrado que os neonatos pré-termo já apresentam as conexões neurais necessárias para experimentar os componentes afetivos da dor no nível cortical (Bartocci et al., 2006; Fitzgerald \& Beggs, 2001; Slater et al., 2006).

Um aumento na concentração total de hemoglobina no córtex somatossensorial contra-lateral foi observado nesses bebês após um procedimento de punção, mas não após uma estimulação tátil não dolorosa, mesmo quando o estímulo sensorial era suficiente para produzir uma resposta motora reflexa (Slater et al., 2006). De acordo com Bartocci et al. (2006), neonatos de 28 a 36 semanas de idade gestacional apresentaram aumento na concentração de hemoglobina em ambos os hemisférios cerebrais e não apresentaram mudanças significativas na frequência cardíaca e na saturação de oxigênio durante a estimulação tátil. A informação nocioceptiva é transmitida ao córtex imaturo do neonato pré-termo e, dessa forma, tem potencial para influenciar níveis superiores do sistema nervoso central em desenvolvimento (Slater et al., 2007), assim como confirma a especificidade da resposta de dor no córtex imaturo.

Levando-se em conta que os procedimentos dolorosos são significativamente freqüentes no tratamento intensivo dos bebês prematuros, avaliar a reação à dor e intervir para minimizá-la são tarefas relevantes e necessárias na implementação de estratégias desenvolvimentais em UTIN (Anand et al., 2006; Gardner \& Lubchenco, 1998). As mudanças na atividade facial, associadas aos estados comportamentais de vigília e sono e aos índices fisiológicos de frequência cardíaca e saturação de oxigênio, tem sido medidas utilizadas como indicadores de dor em bebês acima de 28 semanas de idade gestacional (Morison, Grunau, Oberlander, \& Whitield, 2001). Essas reações para modulação dos estados de alerta fisiológicos e comportamentais fazem parte do processo de autorregulação primária (Calkins, 2009; Morison et al., 2001; Olson, Sameroff, Lunkenheimer, \& Kerr, 2009).

A avaliação da dor em bebês deve ser preferencialmente multidimensional, utilizando tanto medidas fisiológicas quanto comportamentais e envolvendo diferentes parâmetros, como recomendam Craig, Whitfield, Grunau, Linton e Hadjistavropoulos (1993). A escala unidimensional denominada Neonatal Facial Coding System ([NFCS], Grunau \& Craig, 1987; Grunau, Oberlander, Holsti, \& Whitfield, 1998) tem sido amplamente utilizada para a avaliação comportamental de dor em recémnascidos. A atividade facial expressa frente a estímulos dolorosos mostrou correlação significativa com as respostas corticais hemodinâmicas em bebês nascidos prétermo (Slater et al., 2007).

Estudos demonstram que as dores crônicas ou pós-cirúrgicas devem ser manejadas com agentes farmacológicos, enquanto que as dores agudas podem ser manejadas com intervenções não-farmacológicas (Anand \& Whit Hall, 2007). Entre as intervenções não farmacológicas, incluem-se o uso de substâncias adocicadas por via oral, do tipo sacarose (Gaspardo, Chimello, et al., 2008; Stevens et al., 2005), glicose (Chermont, 2008; Gradin \& Schollin, 2005) e frutose (Akçam, 2004), a sucção não nutritiva (Stevens, Yamada, \& Ohlsson, 2001), o contato pele-apele (Castral, Warnock, Leite, Haas, \& Scochi, 2008; Chermont, 2008) e o Método Canguru (Johnston et al., 2009). As intervenções não-farmacológicas para o alívio 
Klein, V. C., Gaspardo, C. M. \& Linhares, M. B. M. (2011). Dor, Autorregulação e Temperamento em Recém-nascidos Pré-termo de Alto Risco.

de dor podem trazer grande benefício ao bebê hospitalizado e têm a sua eficácia comprovada, não apresentando riscos e tendo baixo custo operacional.

\section{Consequências da Exposição Precoce à Dor para o Desenvolvimento}

Devido ao processo de maturação dos sistemas sensoriais que ocorre no período neonatal, este consiste em uma "janela" temporal particularmente sensível para a plasticidade neuronal induzida pela experiência (Hermann, Hohmeister, Demirakça, Zohsel, \& Flor, 2006). A exposição repetida à dor e ao estresse na UTIN pode alterar o substrato neurológico associado com o processamento de dor e, assim, levar a mudanças no processamento somatossensorial da dor e a respostas neurocomportamentais alteradas à dor (Simons \& Tibboel, 2006; Van de Velde et al., 2006).

O fenômeno de repetição do estímulo nocivo pode levar a: (a) hiperalgesia, reposta exagerada a um estímulo que permanece mesmo após cessar o estímulo nocivo original (Taddio, Shah, Atenafu, \& Katz, 2009); (b) alodínia, reações de dor quando manuseado sem estímulo doloroso (Chimello, Gaspardo, Cugler, Martinez, \& Linhares, 2009; Goubet, Clifton, \& Shah, 2001); (c) aprendizagem por associação de estímulos e antecipação de eventos dolorosos (Chimello et al., 2009; Gaspardo, Chimello, et al., 2008; Goubet et al., 2001).

Poucos são os estudos sobre o impacto das experiências de dor do período neonatal no desenvolvimento de crianças nascidas pré-termo (Grunau et al., 2006). O estudo de Grunau et al. (2005) analisou a relação entre exposição à dor no período neonatal e reatividade e encontrou que, em neonatos de idade gestacional menor do que 28 semanas, maior exposição à dor foi significativamente relacionada à menor reatividade facial à dor, assim como à menor nível de cortisol (hormônio marcador biológico de estresse) na $32^{\mathrm{a}}$ semana de idade corrigida para prematuridade. No grupo de idade gestacional maior do que 28 semanas, maior gravidade clínica foi significantemente associada à menor frequência cardíaca. No grupo de menor idade gestacional, após controlar a gravidade clínica e a exposição à morfina, maior exposição à dor neonatal predisse menor nível de cortisol. Além disso, após controlar a gravidade clínica, maior número de procedimentos dolorosos após o nascimento predisse menor atividade facial em resposta à dor.

Em outro estudo, Grunau, Weinberg e Whitfield (2004) encontraram níveis significativamente mais altos de cortisol salivar aos oito meses de idade corrigida para a prematuridade antes e depois da apresentação de um estímulo visual novo no grupo de bebês com idade gestacional extremamente baixa (< 28 semanas) em comparação com os grupos de bebês de idade gestacional muito baixa (2932 semanas) e de bebês a termo. Após controlar a gravidade neonatal e a quantidade de exposição à oxigenação artificial, verificou-se que os maiores níveis de cortisol durante a linha de base em bebês pré-termo aos oito meses foram associados com maior número de procedimentos dolorosos ocorridos no período neonatal. Esses achados demonstram que bebês que sofreram um maior número de procedimentos dolorosos foram mais propensos a apresentar maiores níveis de estresse antes mesmo da apresentação de um estímulo supostamente estressor.

\section{Regulação, Temperamento e Reatividade à Dor no Desenvolvimento dos Recém-Nascidos Pré-termo}

Para a melhor compreensão dos processos envolvidos no interjogo entre fatores de risco e mecanismos de proteção nas trajetórias de desenvolvimento de crianças vulneráveis, é importante se considerar os processos de autorregulação (Feldman, 2009; Sameroff, 2009). A autorregulação envolve processos que amadurecem ao longo do desenvolvimento de maneira contínua, especialmente nos primeiros seis anos de idade da criança, descrito por Olson et al. (2009) da seguinte forma: (a) homeostase fisiológica, que se refere à modulação dos estados fisiológicos de alerta, particularmente como reatividade emocional aos estímulos sensoriais, incluindo duração e intensidade do choro, tempo para se recuperar, capacidade para se acalmar e resposta de cortisol frente a estressores; (b) regulação emocional, que se refere à capacidade de ajustar respostas afetivas, de atenção e de comportamento motor voluntário de forma dirigida ao alcance de objetivos; (c) regulação do comportamento, que se refere a se tornar consciente das demandas sociais e ser capaz de ajustar comportamentos de acordo com essas demandas, devido ao amadurecimento rápido das habilidades cognitivas e motoras; (d) regulação da atenção, que consiste no desenvolvimento do pensamento simbólico ou representacional e da memória, que é subjacente ao aumento da habilidade de adiar a gratificação imediata dos desejos e engajar-se em automonitoramento do comportamento, como consequência da informação que pode ser relembrada; (e) autorregulação, que consiste na capacidade de automonitorar seu comportamento em resposta a diferentes demandas situacionais. Nesta fase, a criança aprende a adaptar-se flexivelmente às situações que possuem diferentes padrões de conduta a elas associados. Emerge o comportamento de autorregulação de discurso internalizado e auto-orientado e estratégias para reduzir a frustração. Esse repertório de respostas de enfrentamento marca a emergência da verdadeira competência de autorregulação.

As capacidades autorregulatórias são fortemente influenciadas pela experiência de regulação oferecida pelos cuidadores da criança (Feldman, 2009; Sameroff, 2009). No início do desenvolvimento, a regulação humana se move de processos biológicos primários para processos psicológicos e sociais. O que começa como um processo de regulação da temperatura, fome e alerta, logo se torna a regulação da atenção, comportamento e interações sociais. Essas aquisições na autorregulação são fortemente 
influenciadas pela regulação do outro. Os pais mantém a criança aquecida, alimentada e acolhida quando esta chora. Os pares (crianças na mesma fase de desenvolvimento) oferecem à criança o conhecimento a respeito dos limites de seu comportamento social; os professores socializam a criança dentro do comportamento do grupo, assim como regulam a cognição em domínios de conhecimento socialmente construídos (Sameroff, 2009).

Dessa forma, o ambiente externo pode constituir-se em desencadeador de mecanismos de proteção no desenvolvimento de crianças nascidas pré-termo por meio de diferentes níveis de regulação externa, denominados micro, mini e macrorregulações, que encontram-se em constante interação e transação (Sameroff, 2009).

De acordo com a classificação proposta por Sameroff (2009), as microrregulações são interações momentâneas que ocorrem entre o indivíduo e os contextos. Ocorrem em um período de tempo muito curto, naturalmente, e são vividas com pouca consciência. São interações momentâneas entre a criança e o cuidador, descritas também como sincronia comportamental. A contribuição da criança para as microrregulações pode ser vista, por exemplo, nos efeitos do temperamento da criança na responsividade materna. As microrregulações consideram o nível individual onde diferenças individuais no temperamento interagem com o comportamento dos cuidadores para produzir uma variedade de relações pais-criança adaptativas e desadaptativas.

No nível das microrregulações, Adam, Gunnar e Tanaka (2004) destacam alguns aspectos da interação pais-criança que atuam como reguladores externos, tais como: (a) a sensibilidade/responsividade calorosa dos pais, que consiste na composição do grau de sensibilidade em que estes respondem às pistas da criança, incluindo a prontidão e adequação de reações, aceitação dos interesses da criança, afeição física, afeto positivo e tom de voz; (b) aceitação calorosa, que se relaciona, no início do desenvolvimento, à afeição física, intimidade, ritmo apropriado e tom de voz positivo, e, em idades posteriores, também inclui uso freqüente de elogios, encorajamento e evitação de comentários negativos para com a criança; (c) responsividade/flexibilidade, que se relaciona, no início do desenvolvimento, à prontidão e adequação de respostas, demonstração de sensibilidade e contingência às pistas da criança e aceitação das necessidades e interesses desta, e, em idades posteriores, também inclui a sustentação materna às explorações da criança e à sua necessidade de independência (Landry et al., 2000); (d) qualidade das direções, definida como a extensão na qual os pais promovem orientação efetiva para a criança sem resolver o problema para ela, fornecendo a esta um senso de controle do mesmo.

Em um nível mais amplo do que as microrregulações, as minirregulações referem-se às atividades dos cuidadores que ocorrem no dia-a-dia, como vestir, alimentar, educar, que variam entre os pais, as famílias e as cultu- ras. As minirregulações operam em um período de tempo mais longo do que as microrregulações. A contribuição da criança para as minirregulações pode ser vista quando os comportamentos do cuidador são reestruturados para atender as demandas específicas da criança, como, por exemplo, demandas de saúde (Sameroff, 2009).

As macrorregulações, por sua vez, são seqüências de marcos no tempo, nos quais o ambiente é reestruturado para proporcionar diferentes experiências para a criança e proporcionam grandes mudanças. Acontecem após intervalos de meses ou anos e variam de cultura para cultura, como a entrada da criança na escola. Os códigos das macrorregulações oferecem a base para a socialização em cada cultura (Sameroff, 2009).

Considerando que o desenvolvimento de aspectos de regulação mais sofisticados e adaptativos pode ser influenciado pelo temperamento da criança em fases iniciais do desenvolvimento (Derryberry \& Rothbart, 2001), tornase importante compreender o temperamento de crianças nascidas pré-termo.

Há evidências acerca da relação entre reatividade do feto (Dipietro, Ghera, \& Costigan, 2008; Dipietro, Hodgson, Costigan, \& Johnson, 1996; Werner et al., 2007) e do bebê (Gunnar, Porter, Wolf, Rigatuso, \& Larson, 1995) e o temperamento em fases posteriores do desenvolvimento. Maior reatividade do feto no terceiro trimestre de gestação foi preditora de maior reatividade motora em resposta a estímulos novos, maior reatividade negativa (Werner et al., 2007), irritabilidade (Dipietro et al., 2008) e choro (Dipietro et al., 1996) na infância. Estes achados enfatizam a tendência constitucional da reatividade inicial e a sua relação com o temperamento.

Tradicionalmente, o temperamento é concebido como diferenças individuais estáveis com forte base genética e neurobiológica (Fox, Henderson, Rubin, Calkins, \& Schmidt, 2001). O conceito de temperamento tem sido integrado crescentemente ao conceito de personalidade. De acordo com Rothbart e Putnam (2002), as diferenças individuais do temperamento constituem a expressão mais precoce da personalidade e o substrato preservado evolutivamente a partir do qual esta se desenvolve. $\mathrm{O}$ temperamento representa o substrato afetivo, de ativação e de atenção da personalidade (Rothbart \& Bates, 2006). Além das disposições temperamentais, a personalidade inclui muitas outras características, tais como auto-conceito, percepção a respeito das outras pessoas, valores pessoais, morais, expectativas, defesas, estratégias de enfrentamento, habilidades, atitudes e crenças. Os traços de temperamento apresentam consistência entre situações e estabilidade ao longo do tempo, mas são limitados aos processos básicos de reatividade e autorregulação e não incluem conteúdos específicos de pensamento ou o uso de defesas, como no caso da personalidade (Rothbart \& Bates, 2006). Esta consiste nos padrões de pensamento e comportamento que apresentam consistência entre situações e estabilidade ao longo do tempo, afetando a adap- 
Klein, V. C., Gaspardo, C. M. \& Linhares, M. B. M. (2011). Dor, Autorregulação e Temperamento em Recém-nascidos Pré-termo de Alto Risco.

tação individual ao mundo interno e ao ambiente social (Rothbart, Ellis, \& Posner, 2004).

De acordo com a abordagem psicobiológica proposta por Rothbart (Rothbart, 1981; Rothbart \& Bates, 2006), o temperamento é entendido como diferenças individuais com base constitucional na reatividade e autorregulação, que são influenciadas ao longo do tempo pela hereditariedade e pela experiência. Reatividade é entendida como características da responsividade individual a mudanças de estimulação externa ou interna apresentada em diversos níveis (comportamental, autonômico, neuroendócrino) e por meio de parâmetros de latência, tempo de aumento, intensidade máxima e tempo de recuperação da reação. A reatividade pode se referir tanto a dimensões mais gerais do comportamento, como reatividade emocional negativa, quanto a reações fisiológicas mais específicas, como a reatividade cardíaca (Rothbart et al., 2004). A reatividade emocional também inclui tendências de ação, de forma que o medo pode produzir imobilidade, ataque e/ou inibição e a afetividade positiva predispõe à aproximação (Rothbart \& Bates, 2006). Os processos de autorregulação têm a função de modular a reatividade comportamental e emocional a fontes de estimulação positiva ou negativa, incluindo comportamentos de aproximação ou retraimento, controle inibitório e controle de atenção.

O temperamento da criança pode ser mensurado por meio de auto-relato (com crianças acima de sete anos de idade), relato dos pais e por meio de observação sistemática em situações estruturadas de laboratório, focalizando o comportamento observado assim como correlatos fisiológicos deste comportamento (Klein, Putnam, \& Linhares, 2009; Rothbart, \& Bates, 2006), como por exemplo frequência cardíaca e nível de cortisol salivar.

Evidências empíricas demonstram que tanto padrões de reatividade podem predizer padrões de temperamento em idades posteriores, quanto padrões de temperamento podem predizer padrões de reatividade posteriormente. Desta maneira, a reatividade à dor envolve aspectos básicos do temperamento (Klein \& Linhares, 2007). Em neonatos a termo, Gunnar et al. (1995) identificaram que maior reatividade fisiológica e comportamental em resposta ao estímulo doloroso foi relacionada a temperamento, com menos angústia frente a limitações aos seis meses de idade cronológica. Em crianças nascidas a termo, o temperamento "não ajustado", indicativo de humor negativo, retraimento e comportamento pouco adaptável, e a experiência prévia de dor foram preditores de reatividade à dor durante uma situação de vacinação aos cinco anos de idade (Rocha, Prkachin, Beaumont, Hardy, \& Zumbo, 2003). O temperamento "não ajustado" da criança e a maior reatividade à dor foram preditores de queixas somáticas nas crianças aos sete anos de idade (Rocha \& Prkachin, 2007).

Em crianças nascidas pré-termo, Klein, Gaspardo, Martinez, Grunau e Linhares (2009) identificaram que maior reatividade biocomportamental à dor e menor regulação nos primeiros dez dias de idade cronológica foram preditores de temperamento com alto afeto negativo e extroversão, na fase dos três primeiros anos de idade cronológica (18-35 meses), após controlar a quantidade de exposição à dor e a gravidade clínica neonatal. O estudo de Klein (2009) demonstrou que, em crianças nascidas pré-termo, maior reatividade fisiológica ao estresse, antes do procedimento doloroso nos primeiros dez dias de idade cronológica, foi preditora de temperamento com maior nível de desconforto na idade pré-escolar (37-57 meses). Dessa maneira, os resultados desses dois estudos indicam que os recursos individuais de autorregulação fisiológica e comportamental do neonato pré-termo tiveram maior peso na explicação das variações no temperamento posterior do que o número de procedimentos dolorosos ao qual o neonato foi submetido. Esses achados contribuíram para elucidar que, em relação ao temperamento, não é a quantidade de exposição a estímulos dolorosos que apresenta efeito sobre os processos de temperamento, mas sim, a maneira com que os neonatos fisiologicamente imaturos reagem e se regulam frente a esses estímulos.

O temperamento de um grupo de crianças nascidas pré-termo na fase dos três primeiros anos, comparado com um grupo de crianças nascidas a termo, foi objeto do estudo de Klein (2009). Nessa fase, crianças nascidas pré-termo apresentaram temperamento com mais inquietação e movimentos repetidos de motricidade fina, mais sensibilidade sensorial, mais prazer relacionado com situações com alta intensidade de estímulos e menos prazer em aconchegar-se ao cuidador do que crianças nascidas a termo. As diferenças individuais nos processos de regulação nos níveis fisiológico e comportamental influenciam a formação da personalidade e o comportamento ajustado durante a primeira infância, quando a autorregulação emocional e comportamental se torna a base para a adaptação bem sucedida (Calkins, 2009). Dessa forma, é importante compreender o desenvolvimento da criança pré-termo no contexto da autorregulação e da regulação externa, considerando as características individuais de temperamento.

\section{Implicações Práticas para o Desenvolvimento da Criança}

A imaturidade dos sistemas do organismo do neonato pré-termo constitui-se uma vulnerabilidade inicial para dificuldades nos processos regulatórios nesse período. Além disso, a exposição à dor e estresse de forma repetida e por um longo período de tempo pode acentuar essas dificuldades. A intervenção preventiva para problemas de desenvolvimento nessa população tem espaço desde as primeiras experiências de autorregulação do bebê a partir de seu nascimento, no nível das microrregulações (Sameroff, 2009). Os profissionais da equipe de saúde 
no contexto da UTIN assumem o papel de cuidador primário do neonato pré-termo, podendo modular as respostas dos neonatos no nível comportamental, emocional, neuroendocrinológico e parassimpático. Esta modulação da autorregulação do neonato por parte dos profissionais da equipe de saúde pode ocorrer por meio do uso de estratégias de alívio de dor e estresse, como, por exemplo, a administração de substâncias adocicadas como a sacarose (Gaspardo, Myiase, Chimello, Martinez, \& Linhares, 2008) e o contato pele-a-pele com a mãe (Castral et al., 2008). Essas estratégias promovem a redução da reatividade biocomportamental do neonato e podem ser um exemplo de provisão, por parte do ambiente, de reguladores escondidos (hidden regulators), os quais, em uma situação de nascimento a termo e sem problemas de saúde, são providos pelo cuidador primário, especialmente a mãe (Calkins, 2009). Os reguladores escondidos operam em múltiplos níveis sensoriais (olfativo, tátil e oral) e podem influenciar múltiplos níveis de funcionamento comportamental e fisiológico no bebê. Oportunidades para diferenças individuais no desenvolvimento da regulação da emoção podem emergir de diferentes condições de cuidado que oferecem maior ou menor regulação psicobiológica para o bebê (Calkins, 2009).

Considerando que a definição de temperamento afirma que este pode ser influenciado pela hereditariedade, maturação e experiência (Derryberry \& Rothbart, 2001), é importante salientar que os pais são uma variável importante no desenvolvimento do temperamento. No nível das minirregulações (Sameroff, 2009), a intervenção preventiva assume um papel em orientar os pais a respeito de como identificar e modular os padrões de autorregulação do bebê, de modo a promover interações sincrônicas pais-criança, especialmente até a idade préescolar.

Após a alta do hospital, os pais e os familiares assumem a função de reguladores externos primários da reatividade biocomportamental do bebê pré-termo. Torna-se relevante o acompanhamento do desenvolvimento de crianças nascidas pré-termo em seus diferentes marcos desenvolvimentais por profissionais especializados em Psicologia Pediátrica, conforme recomendado por Linhares, Carvalho, Correia, Gaspardo e Padovani (2006). No programa de follow-up, devem-se identificar as características de autorregulação, temperamento e comportamento da criança; dessa forma, é possível orientar os pais e ajudá-los a compreender essas diferenças individuais e dar suporte para que eles aprendam a manejá-las.

Orientações sistematizadas dirigidas aos pais, a fim de promover percepções mais realísticas em relação aos processos de autorregulação e ao temperamento da criança, podem aumentar a sensibilidade e a responsividade dos pais durante a interação, promovendo a autorregulação da criança nascida pré-termo. Com este enfoque, as orientações assumem um caráter de intervenção preventiva, permitindo evitar a ocorrência de problemas de comporta- mento ao longo do desenvolvimento e, consequentemente, protegendo a saúde mental dessas crianças em risco.

\section{Considerações Finais}

Conclui-se que: (a) os neonatos pré-termo sentem dor; (b) a dor pode ser avaliada; (c) a dor neonatal exerce impacto negativo no desenvolvimento; (d) o mecanismo de reatividade-regulação do organismo pode ser afetado ao longo do desenvolvimento; (e) o temperamento da criança vulnerável pode agravar ou proteger o seu desenvolvimento; (f) existem intervenções eficazes para prevenção e alívio da dor e estresse, a fim de corregular o bebê imaturo e vulnerável; (g) programas de prevenção devem incluir a orientação de pais sobre como promover a regulação da criança nascida pré-termo por meio das interações sociais, caracterizadas por responsividade, calorosidade e sensibilidade parental.

\section{Referências}

Adam, E. K., Gunnar, M. R., \& Tanaka, A. (2004). Adult attachment, parent emotion, and observed parenting behavior: Mediator and moderator models. Child Development, 75(1), 110-122.

Akçam, M. (2004). Oral fructose solution as an analgesic in the newborn: A randomized, placebo-controlled and masked study. Pediatrics International, 46(4), 459-462.

Als, H., Duffy, F. H., Mcanulty, G. B., Rivkin, M. J., Vajapeyam, S., Mulkern, R. V., et al. (2004). Early experience alters brain function and structure. Pediatrics, 113(4), 846-857.

Anand, K. J. S., Aranda, J. V., Berdec, B., Buckman, S., Capparelli, E. V., Carlo, W., et al. (2006). Summary proceedings from the neonatal pain-control group. Pediatrics, $117,9-22$.

Anand, K. J. S., \& Whit Hall, R. W. (2007). Controversies in neonatal pain: An introduction. Seminars in Perinatology, 31(5), 273-274.

Barbosa, V. C., Formiga, C. K. M. R., \& Linhares, M. B. M. (2007). Avaliação das variáveis clínicas e neurocomportamentais de recém-nascidos pré-termo. Revista Brasileira de Fisioterapia, 11(4), 275-281.

Bartocci, M., Bergqvist, L. L., Lagercrantz, H., \& Ananda, K. J. (2006). Pain activates cortical areas in the preterm newborn brain. Pain, 122, 109-117.

Calkins, S. (2009). Regulatory competence and early disruptive behavior problems: The role of physiological regulation. In S. L. Olson \& A. J. Sameroff (Eds.), Biopsychosocial regulatory processes in the development of childhood behavioral problems (pp. 86-107). New York: Cambridge University Press.

Castral, T. C., Warnock, F., Leite, A. M., Haas, V. J., \& Scochi, C. G. (2008). The effects of skin-to-skin contact during acute pain in preterm newborns. European Journal of Pain, 12(4), 464-471.

Chermont, A. G. (2008). Glicose e contato pele-a-pele para redução da dor aguda em recém-nascidos a termo: Ensaio clínico randomizado e controlado. Tese de Doutorado nãopublicada, Escola Paulista de Medicina, Universidade Federal de São Paulo, SP. 
Chimello, J. T., Gaspardo, C. M., Cugler, T. S., Martinez, F. E., \& Linhares, M. B. M. (2009). Pain reactivity and recovery in preterm neonates: Latency, magnitude, and duration of behavioral responses. Early Human Development, 85(5), 313318.

Clark, C. A. C., Woodward, L. J., Horwood, L. J., \& Moor, S. (2008). Development of emotional and behavioral regulation in children born extremely preterm and very preterm: Biological and social influences. Child Development, 79, 1444-1462.

Craig, K. D., Whitfield, M. F., Grunau, R. V. E., Linton, J. E., \& Hadjistavropoulos, H. D. (1993). Pain in the preterm neonate: Behavioral and physiological indices. Pain, 52(3), 287-299.

Couperus, J. W., \& Nelson, C. A. (2006). Early brain development and plasticity. In K. McCartney \& D. Phillips (Eds.), Blackwell Handbook of early childhood development (pp. 85-105). Malden, MA: Blackwell.

Derryberry, D., \& Rothbart, M. K. (2001). Early temperament and emotional development. In A. F. Kalverboer \& A. Gramsbergen (Eds.), Handbook of brain and behavior in human development (pp. 967-987). Dordrecht, Netherlands: Kluwer Academic.

Dipietro, J. A., Ghera, M. M., \& Costigan, K. A. (2008). Prenatal origins of temperamental reactivity in early infancy. Early Human Development, 84, 569-575.

Dipietro, J. A., Hodgson, D. M., Costigan, K. A., \& Johnson, T. R. B. (1996). Fetal antecedents of infant temperament. Child Development, 67, 2568-2583.

Feldman, R. (2009). The development of regulatory functions from birth to 5 years: Insights from premature infants. Child Development, 80(2), 544-561.

Fitzgerald, M., \& Beggs, S. (2001). The neurobiology of pain: Developmental aspects. The Neuroscientist, 7(3), 246-257.

Fox, N. A., Henderson, H. A., Rubin, K. H., Calkins, S., D., \& Schmidt, L. A. (2001). Continuity and discontinuity of behavioral inhibition and exuberance: Psychophysiological and behavioral influences across the first four years of life. Child Development, 72(1), 1-21.

Franck, L., \& Miakowski, C. (1997). Measurement of neonatal responses to painful stimuli: A research review. Journal of Pain and Symptom Management, 14(96), 343-378.

Gardner, S., \& Lubchenco, L. O. (1998). The neonatal and the environment: Impact on development. In G. B. Merenstein \& S. L. Gardner (Eds.), Handbook of neonatal intensive care (pp. 197-241). Toronto, Canada: Mosby.

Gaspardo, C. M., Chimello, J. T., Cugler, T. S., Martinez, F. E., \& Linhares, M. B. M. (2008). Pain and tactile stimuli during arterial puncture in preterm neonates. Pain, 140, 58-64.

Gaspardo, C. M., Miyase, C. I., Chimello, J. T., Martinez, F. E., \& Linhares, M. B. M. (2008). Is pain relief equally efficacious and free of side effects with repeated doses of oral sucrose in preterm neonates? Pain, 137, 16-25.

Glass, P. (1999). O recém-nascido vulnerável e o ambiente na unidade de tratamento intensivo neonatal. In G. B. Avery, M. A. Fletcher, \& M. G. MacDonald (Eds.), Neonatologia: Fisiopatologia e tratamento do recém-nascido (pp. 79-96). São Paulo, SP: MEDSI.

Goubet, N., Clifton, R. K., \& Shah, B. (2001). Learning about pain in preterm newborns. Journal of Developmental and Behavioral Pediatrics, 22(6), 418-424.

Gradin, M., \& Schollin, J. (2005). The role of endogenous opioids in mediating pain reduction by orally administered glucose among newborns. Pediatrics, 115(4), 1004-1007.
Grunau, R. V. E., \& Craig, K. D. (1987). Pain expression in neonates: Facial action and cry. Pain, 28, 395-410.

Grunau, R. V. E., Holsti, L., Haley, D. W., Oberlander, T., Weinberg, J., Solimano, A., et al. (2005). Neonatal procedural pain exposure predicts lower cortisol and behavioral reactivity in preterm infants in the NICU. Pain, 113(3), 293-300.

Grunau, R. V. E., Holsti, L., \& Peters, J. W. B. (2006). Longterm consequences of pain in human neonates. Seminars in Fetal \& Neonatal Medicine, 11, 268-275.

Grunau, R. V. E., Oberlander, T., Holsti, L., \& Whitfield, M. F. (1998). Bedside application of the Neonatal Facial Coding System in pain assessment of premature neonates. Pain, 76, 277-286.

Grunau, R. V. E., Weinberg, J., \& Whitfield, M. (2004). Neonatal procedural pain and preterm infant cortisol response to novelty at 8 months. Pediatrics, 114(1), 77-84.

Gunnar, M. R., Porter, F. L., Wolf, C. M., Rigatuso, J., \& Larson, M. C. (1995). Neonatal stress reactivity: Predictions to later emotional temperament. Child Development, 66, 1-13.

Hermann, C., Hohmeister, J., Demirakça, S., Zohsel, K., \& Flor, H. (2006). Long-term alteration of pain sensitivity in schoolaged children with early pain experiences. Pain, 125, 278-285.

Johnston, C. C., Filion, F., Campbell-Yeo, M., Goulet, C., Bell, L., McNaughton, K., et al. (2009). Enhanced kangaroo mother care for heel lance in preterm neonates: A crossover trial. Journal of Perinatology, 29(1), 51-56.

Klein, V. C. (2009). Reatividade à dor, temperamento e comportamento na trajetória de desenvolvimento de neonatos pré-termo até a fase pré-escolar. Tese de Doutorado nãopublicada, Faculdade de Medicina, Universidade de São Paulo, Ribeirão Preto, SP.

Klein, V. C., Gaspardo, C. M., Martinez, F. E., Grunau, R. E., \& Linhares, M. B. M. (2009). Pain and distress reactivity and recovery as early predictors of temperament in toddlers born preterm. Early Human Development, 85(9), 569-576.

Klein, V. C., \& Linhares, M. B. M. (2006). Prematuridade e interação mãe-criança: Revisão sistemática da literatura. Psicologia em Estudo, 11, 284-294.

Klein, V. C., \& Linhares, M. B. M. (2007). Temperamento, comportamento e experiência dolorosa na trajetória de desenvolvimento da criança. Cadernos de Psicologia e Educação - Paidéia, 17(36), 33-44.

Klein, V. C., Putnam, S. P., \& Linhares, M. B. M. (2009). Assessment of temperament in children: Translation of instruments to Portuguese (Brazil) Language. Interamerican Journal of Psychology, 43(3), 442-447.

Landry, S. H., Smith, K. E., Swank, P. R., \& Miller- Loncar, C. L. (2000). Early maternal and child influences on children's later independent cognitive and social functioning. Child Development, 71(2), 358-375.

Laucht, M., Esser, G., \& Schmidt, M. H. (2002). Vulnerability and resilience in the development of children at risk: The role of early mother-child interaction. Revista de Psiquiatria Clínica, 29(1), 20-27.

Linhares, M. B. M. (2009). Vulnerabilidade, risco e proteção no desenvolvimento de crianças nascidas pré-termo. Tese de Livre-Docência não-publicada, Faculdade de Medicina, Universidade de São Paulo, Ribeirão Preto, SP.

Linhares, M. B. M., Martins, I. M. B., \& Klein, V. C. (2004). Mediação materna como processo de promoção e proteção do desenvolvimento da criança nascida prematura. In E. M. Marturano, M. B. M. Linhares, \& S. R. Loureiro (Eds.), Vulnerabilidade e proteção: Indicadores na trajetória de desenvolvimento do escolar (pp. 39-74). São Paulo, SP: Casa do Psicólogo. 
Linhares, M. B. M., Carvalho, A. E. V., Correia, L. L., Gaspardo, C. M., \& Padovani, F. H. P. (2006). Psicologia Pediátrica em neonatologia de alto risco: Promoção precoce do desenvolvimento de bebês prematuros. In M. A. Crepaldi, M. B. M. Linhares, \& G. B. Perosa (Eds.), Psicologia Pediátrica (pp. 109-146), São Paulo, SP: Casa do Psicólogo.

Lorenz, J. M. (2000). Survival of the extremely preterm infant in North America in the 1990s. Clinics of Perinatology, 27(2), 255-262.

Luthar, S. S., Sawyer, J. A., \& Brown, P. J. (2006). Conceptual issues in studies of resilience: Past, present, and future research. Annals of New York Academy of Sciences, 1094, 105-115.

Luu, T. M., Ment, L. R., Schneider, K. C., Katz, K. H., Allan, W. C., \& Vohr, B. R. (2009). Lasting effects of preterm birth and neonatal brain hemorrhage at 12 years of age. Pediatrics, 123, 1037-1044.

Méio, M. D. B. B., Lopes, C. S., \& Morsch, D. S. (2003). Fatores prognósticos para o desenvolvimento cognitivo de prematuros de muito baixo peso. Revista de Saúde Pública, 37, 311-318.

Minde, K. (2000). Prematurity and serious medical conditions in infancy: Implications for development, behavior and intervention. In C. Zeanah (Ed.), Handbook of infant mental health (pp. 176-194). New York: The Guilford Press.

Ministério da Saúde. (2009, fev.). Cadernos de Informações de saúde. Retrieved April 28, 2010, from http://tabnet.datasus. gov.br/tabdata/cadernos/cadernosmap.htm\#cadernos

Morison, S., Grunau, R., Oberlander, T., \& Whitield, M. (2001). Relationships between behavioral and cardiac autonomic reactivity to acute pain in preterm infants. The Clinical Journal of Pain, 17(4), 350-358.

Olson, S. I., Sameroff, A., Lunkenheimer, E. S., \& Kerr, D. (2009). Self-regulatory processes in the development of disruptive behavior problems: The preschool to school transition. In S. L. Olson \& A. J. Sameroff (Eds.), Biopsychosocial regulatory processes in the development of childhood behavioral problems (pp. 144-185). New York: Cambridge University Press.

Rocha, E. M., \& Prkachin, K. M. (2007). Temperament and pain reactivity predict health behavior seven years later. Journal of Pediatric Psychology, 32(4), 393-399.

Rocha, E. M., Prkachin, K. M., Beaumont, S. L., Hardy, C. L., \& Zumbo, B. D. (2003). Pain reactivity and somatization in kindergarten-age children. Journal of Pediatric Psychology, 28(1), 47-57.

Rothbart, M. K. (1981). Measurement of temperament in infancy. Child Development, 52(2), 569-578.

Rothbart, M. K., \& Bates, J. E. (2006). Temperament. In W. Damon, R. M. Lerner (Series Ed.), \& N. Eisenberg (Vol. Ed.), Handbook of Child Psychology: Vol. 3. Social, emotional and personality development ( $5^{\text {th }}$ ed., pp. 99-165). New York: John Wiley and Sons.

Rothbart, M. K., Ellis, L. K., \& Posner, M. I. (2004). Temperament and self-regulation. In R. F. Baumeister \& K. D. Vohs (Eds.), Handbook of self-regulation: Research, theory, and applications (pp. 357-370). New York: Guilford Press.

Rothbart, M. K., \& Putnam, S. (2002). Temperament and socialization. In L. Pulkinnem \& A. Caspi (Eds.), Paths to successful development: Personality in the life course (pp. 19-45). Cambridge, MA: Cambridge University Press.
Rutter, M. (2006). Implications of resilience concepts for scientific understanding. Annals of New York Academy of Sciences, 1094, 1-12.

Salt, A., \& Redshaw, M. (2006). Neurodevelopmental followup after preterm birth: Follow up after two years. Early Human Development, 82, 185-197.

Samara, M., Marlow, N., \& Wolke, D. (2008). Pervasive behavior problems at 6 years of age in a total-population sample of children born at $<25$ weeks of gestation. Pediatrics, 122, 562-573.

Sameroff, A. J. (2009). Conceptual issues in studying the development of self-regulation. In S. L. Olson \& A. J. Sameroff (Eds.), Biopsychosocial regulatory processes in the development of childhood behavioral problems (pp. 1-18). New York: Cambridge University Press.

Simons, S. H. P., \& Tibboel, D. (2006). Pain perception development and maturation. Seminars in Fetal \& Neonatal Medicine, 11, 227-231.

Slater, R., Cantarella, A., Gallella, S., Worley, A., Boyd, S., Meek, J., et al. (2006). Cortical pain responses in human infants. The Journal of Neuroscience, 26(14), 3662-3666.

Slater, R., Fitzgerald, M., \& Meek, J. (2007). Can cortical responses following noxious stimulation inform us about pain processing in neonates? Seminars in Perinatology, 31(5), 298-302.

Stevens, B., Yamada, J., Beyene, J., Gibbins, S., Petryshen, P., Stinson, J., et al. (2005). Consistent management of repeated procedural pain with sucrose in preterm neonates: Is it effective and safe for repeated use over time? The Clinical Journal of Pain, 21, 543-548.

Stevens, B., Yamada, J., \& Ohlsson, A. (2001). Sucrose for analgesia in newborn infants undergoing painful procedures [CD-ROM]. Cochrane Database of Systematic Reviews, 4, CD 001069.

Sun, J., Mohay, H., \& O’Callaghan, M. A. (2008). Comparison of executive function in very preterm and term infants at eight months corrected age. Early Human Development, 85, 225-230.

Taddio, A., Shah, V., Atenafu, E., \& Katz, J. (2009). Influence of repeated painful procedures and sucrose analgesia on the development of hyperalgesia in newborn infants. Pain, 144(12), 43-48.

Van de Velde, M. C., Jani, J., De Buck, F., \& Deprest, J. (2006). Fetal pain perception and pain management. Seminars in Fetal and Neonatal Medicine, 11, 232-236.

Werner, E. A., Myers, M. M., Fifer, W. P., Cheng, B., Fang, Y., Allen, R., et al. (2007). Prenatal predictors of infant temperament. Developmental Psychobiology, 49(5), 474-484.

Wolke, D., Samara, M., Bracewell, M., \& Marlow, N. (2008). Specific language difficulties and school achievement in children born at 25 weeks of gestation or less. The Journal of Pediatrics, 15(2), 256-262.

Yunes, M. A., \& Szymanski, H. (2001). Resiliência: Noção, conceitos afins e considerações críticas. In J. Tavares (Ed.), Resiliência e Educação (pp. 13-42). São Paulo, SP: Cortez. 